\title{
Tropospheric Scatter Propagation and Atmospheric Circulations
}

\author{
William F. Moler ${ }^{1}$ and D. B. Holden ${ }^{1}$
}

(August 10, 1959)

\begin{abstract}
Transhorizon vhf and uhf fields exhibit deep fades or large signal enhancements of several hours' duration, as the propagation mechanism alternates between partial reflection and scattering caused by turbulent dielectric fluctuations in the atmosphere. Such alternations occur when strong refractive layers develop below 3,000 ft. Surface wind streamline analyses show that mesoscale centers of convergence or divergence cause local redistribution of refractive layering, tending to produce the change from one mechanism to the other.

Current scattering theory and the empirical findings of others are examined to determine the gross meteorological factors that influence changes in scattered fields. The two variables in the turbulent scattering coefficients, the scattering angle and the intensity of dielectric fluctuations at high wave numbers, are found to be dependent upon the refractive layering and the thermal stability of the airmass. It has been shown elsewhere that refractivity and stability are principally functions of the vertical velocity in the atmosphere. It is shown here that the direction and relative magnitude of the vertical velocity can be inferred from the upper-tropospheric wind velocity divergence. Received scattered signals are found to be well correlated with computed velocity divergence.

It is suggested that the variations of scattered signal level or range can be predicted in a routine manner by regular meteorological personnel using ordinarily available meteorological data.
\end{abstract}

\section{Introduction}

Future Navy communication networks and early warning radar may depend to a great extent upon the tropospheric scatter mode of radio propagation. The development of high-gain low-noise receivers and statistical target identification techniques makes the vhf and uhf region of the radio spectrum useful for ranges well beyond the radio horizon.

The signal in the far transhorizon region is characterized by a rapidly fluctuating power amplitude superimposed on a more slowly changing average signal level. The rate of fast fading depends on distance and radiofrequency, indicating the existence of tuncorrelated multipath transmission from numerous moving scattering centers. The slow fading has seasonal, average diurnal, and random day-today or hourly components. Seasonal and average diurnal fluctuations are amenable to statistical analysis and are predictable within relatively narrow limits) [1]. ${ }^{2}$ The random daily or hourly variations in signal level have been less well understood. These are the fluctuations in signal level (or range) in which the Navy may be most interested.

The best current theories explain the scatter propagation mechanism by making use of scattering coefficients which are functionally related to the gross refractivity of the lower atmosphere and the intensity of turbulent dielectric fluctuations at radiofrequencydependent scale sizes. The scattering coefficients are difficult to measure and, at the present time, are unpredictable. It is possible to predict some aspects of the atmosphere's refractive structure which, from elementary considerations of theory, are most likely

1 U.S. Navy Electronics Laboratory, San Diego 52, Calif.

2 Figures in brackets indicate the literature references at the end of this paper. to control the magnitude of the scattering coefficients and hence the transhorizon signal strength.

This report indicates the meteorological factors which appear to control the electromagnetic scattering mechanism and suggests an operational method for predicting the direction and relative magnitude of signal level change.

\section{Discussion of Theoretical and Fmpirical Relationships}

The theories proposed to explain the large otherthan-diffraction transhorizon fields are based on two propagation mechanisms: (1) partial reflection of incident radiation by horizontally stratified dielectric layers $[2,3,4]$, and (2) scattering by turbulent discontinuities in the dielectric structure of the atmosphere $[5,6,7]$. The phenomenon of partial reflection has been recognized and studied for over ten years. In the microwave region, for usual refractive gradients, the effect of layers decreases exponentially with height, so that reflections of this sort become unimportant for layers above about $3,000 \mathrm{ft}$. More recently, Bauer, Friis, et al. [8, 9] theorized that energy may be partially reflected in much the same manner from thin, highly refractive sublamina within the region illuminated by both antennas. Refractometer measurements made during convective atmospheric conditions by Navy Electronics Laboratory (NEL) failed to show the existence of such layering at times when transhorizon radio transmission was normal. It is likely that, under conditions of a well-established elevated layer, both propagation mechanisms may exist concurrently [10], but that in the absence of strong relatively low elevated layers, the turbulent scattering mechanism is dominant. 
The turbulent scattering theories, following the original work of Booker and Gordon [5], postulate in common an equation of the general form:

$$
P r \propto \frac{1}{\sin ^{n} \frac{\theta}{2}} F(K) .
$$

$\operatorname{Pr}=$ received power,

$\theta=$ intersection angle of rays from transmitter and receiver,

$$
K=\frac{4 \pi \sin \frac{\theta}{2}}{\lambda},
$$

$\lambda=$ radio wavelength,

$F(K)=$ function of dielectric fluctuation intensity at wave number, $k$, and

$n=$ exponent, dependent on form of $F$.

By incorporating the modern theories of turbulence put forth by Obukhoff, Kolmogoroff, and others with radio scattering theory, Batchelor [7] found that, for an isotropic radiator, $n$ has the value $11 / 3$. For propagation with narrow beam antennas, the exponent becomes $14 / 3$. It should be noted that for grazing propagation and ducting gradients, expression (1) fails; propagation is line-of-sight and, disregarding focusing effects, power is maximized.

\subsection{Scattering Angle}

The strong dependence of signal level upon scattering angle (mean refractive gradients) is demonstrated in figure 1. The abscissa is the signal level

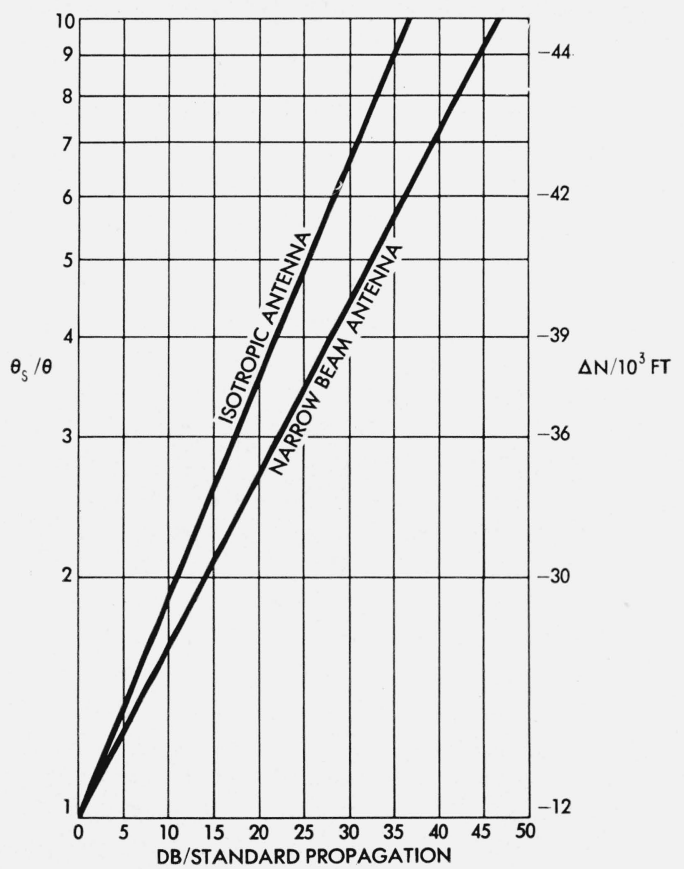

FIGURE 1. Scattered field signal level as a function of scattering angle and refractive gradients in a homogeneous refractive atmosphere normalized to standard gradient. in decibels above that received in a standard atmosphere ( $-12 N$ units $/ 10^{3} \mathrm{ft}$ ) for ratios of the standard atmosphere scattering angle to the actual scattering angle (ordinate on the left) given by the $N$ gradients (along the right of the graph). It is noteworthy that with high signal levels and near-ducting gradients, deep fades usually associated with other modes of propagation can occur with very slight changes in the low level refractive gradients.

A method for determining the scattering angle from the refractive index profiles over the scatter propagation link is outlined in appendix $\mathrm{A}$. The method is generalized for a multilayered atmosphere, the layers having a limited degree of horizontal homogeneity.

Other investigators concerned with developing methods for predicting transhorizon fields through use of meterorological parameters have found correlation between scattered fields and low-level refractive gradients. Bean [1], reasoning that lower tropospheric refractive gradients are directly related to the surface value of refractive index, found statistical correlation between transhorizon fields and the surface refractivity. Misme [11], using a weighted average tropospheric refractivity, found correlation between this "useful gradient" and the received signal.

Time-averaged low-level refractive gradients have been shown by Moler and Arvola [12], to be principally a function of the large-scale vertical motions in the atmosphere. Later in this report, computations of convergence at the 300-millibar (mb) level and the implied subsidence (see app. B) throughout the troposphere are compared with the development and descent of a strong refractive layer and the resultant change in scattering angle and radio signal strength.

Low-level gradients may also be temporarily altered with the development of minor eddies in the surface wind flow. Occasionally, during the NEL scatter propagation experiments, radio signal levels and characteristics would indicate a change in the propagation mechanism from scattering to partial reflections. Subsequent analysis of the smallscale wind circulations showed that local regions of convergence (divergence) which develop within a relatively homogeneous large-scale weather regime cause raising (lowering) of refractive layers and, consequently, a change from one dominant mode of propagation to another. Examples of this will be given in a later section of this report.

\subsection{Turbulent Dielectric Fluctuations}

Little is known about the variation of intensity of turbulent dielectric fluctuations at wave numbers important for microwave scattering as a function of the meterorological situation. Recent work by Gossard [13] indicated that the refractive index spectral intensity at high wavenumbers in the inertial subrange of turbulence is only weakly dependent upon atmospheric stability or refractive gradients. From this work, it may be concluded that variations 
in signal level result almost entirely from refractive changes in scattering angle or that there are thin zones of quite variable turbulent intensity which superimpose an amplitude modulation upon a low average signal.

Batchelor [7] suggested that the fluctuations of refractive index may be due to turbulent convection in the presence of a spatial gradient of mean refractive index. If this suggestion is correct, it seems likely that the greatest intensity of dielectric fluctuation would occur along the lower boundary of elevated stable layers (refractive layers) where convective energy from the lower, less stable region is absorbed and dissipated.

Based on empirical evidence, Misme [11] found that "for equal refractive index gradients, the atmosphere which is characterized by the greatest stability permits the highest fields to be received from a great distance." By "equal gradients", he meant the refractivity which determines the scattering angle.

Gordon [14], using assumed values of fluctuation intensity, concluded that the contribution of scattered energy from a single turbulent layer may be greater than that from the remaining common volume. The theories of partial reflection $[2,3,4$, $8,9]$ require the presence of highly refractive substrata within a mean refractive gradient with greatest radio fields associated with intense elevated refractive layers.

All evidence indicates that departures of radio fields from a low ambient level can best be forecast indirectly through a prediction of the atmospheric thermal stability. It is well known that, in general, atmospheric stability is largely a function of the vertical components of the winds. At the onset of subsidence, refractive layers develop; continuous subsidence causes lowering and intensification of the layers and increases the thermal stability of the airmass. Rising motion tends first to elevate the layers and later to cause their complete dissipation.

The behavior of radio signal levels under the influence of positive as opposed to negative vertical motion will be shown in a later section. The method for deducing the sign and relative magnitude of the vertical wind component from upper tropospheric constant pressure maps will be found in appendix B.

\section{Operations}

In January 1958, NEL activated three transhorizon radio links which were in periodic operation until mid-April. Stations at Santa Barbara (190 statute miles) and at Point Mugu (144 statute miles) transmitted $9,380 \mathrm{Mc}$ ( $X$-band) and $1,365 \mathrm{Mc}(L-$ band) pulsed signals. A third station on San Clemente Island (78 statute miles) transmitted at 9,380 Mc only. All receivers were located on Point Loma, San Diego. Figure 2 is a map of the southern California operating area. Signal levels used in this report were measured over 1-min intervals on integrating equipment and recorded in decibels with respect to 1 milliwatt.

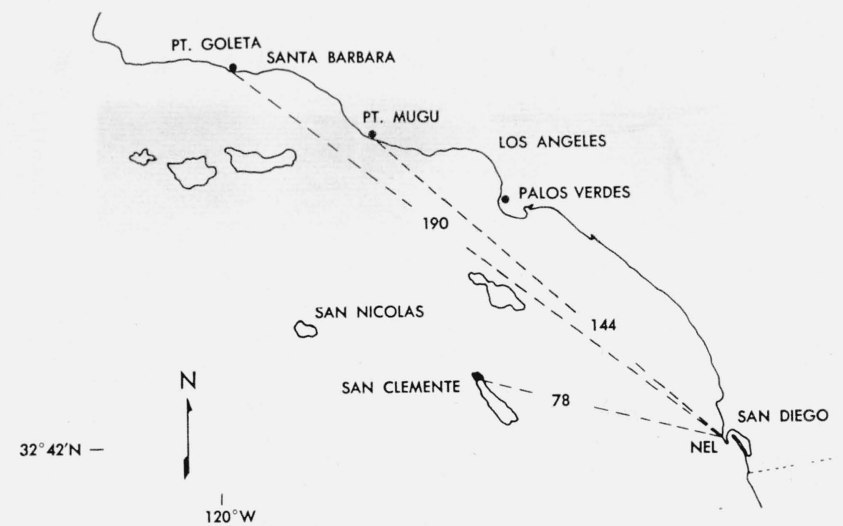

Figure 2. Navy Electronics Laboratory scatter propagation experiment operations area.

Strong low-level elevated refractive layers are a dominant feature along the southern California coast and frequently cause ducting or abnormally strong signal enhancement from internal reflections when the layers are lower than about $3,000 \mathrm{ft}[3,4]$. In order to avoid such conditions, the links were placed in operation only when there was a reaonable assurance that refractive layers would be absent or at a high elevation. Synoptic experience has shown that airmasses are most unstable and thermal layering absent when under the influence of a strong cyclonic circulation through most of the troposphere. For this reason, the bulk of data was taken during periods when a deep low pressure system at the $500-\mathrm{mb}$ level $(18,000 \mathrm{ft})$ was near or over the radio link.

Radio link operations were discontinued whenever signal characteristics indicated that a propagation mode other than scattering was dominant and likely to continue. Unfortunately, as a result very little data were taken during periods of high signal level and conditions of airmass subsidence.

The southern California sectional maps were constructed from hourly airways teletype reports and from weather logs of two aircraft carriers which were operating in coastal waters. The surface synoptic and $500-\mathrm{mb}$ charts are copies of National Weather Analysis Center maps transmitted over the facsimile network. Calculations of vorticity advection at the 300-mb levels were made from maps plotted at NEL, using data from the Daily Bulletin of Northern Hemisphere Data Tabulations published by the U.S. Weather Bureau.

\section{Measurements}

\subsection{Signal Level and Refractive Layering}

The constant pressure contours for the $300-\mathrm{mb}$ pressure surface for 1600 P.s.t., March 16 to 19 , are shown in figure 3 . The field of relative vorticity (dashed lines) indicates positive vorticity advection (horizontal divergence) east of the upper level trough and negative vorticity advection (horizontal convergence) west of the trough. From continuity con- 

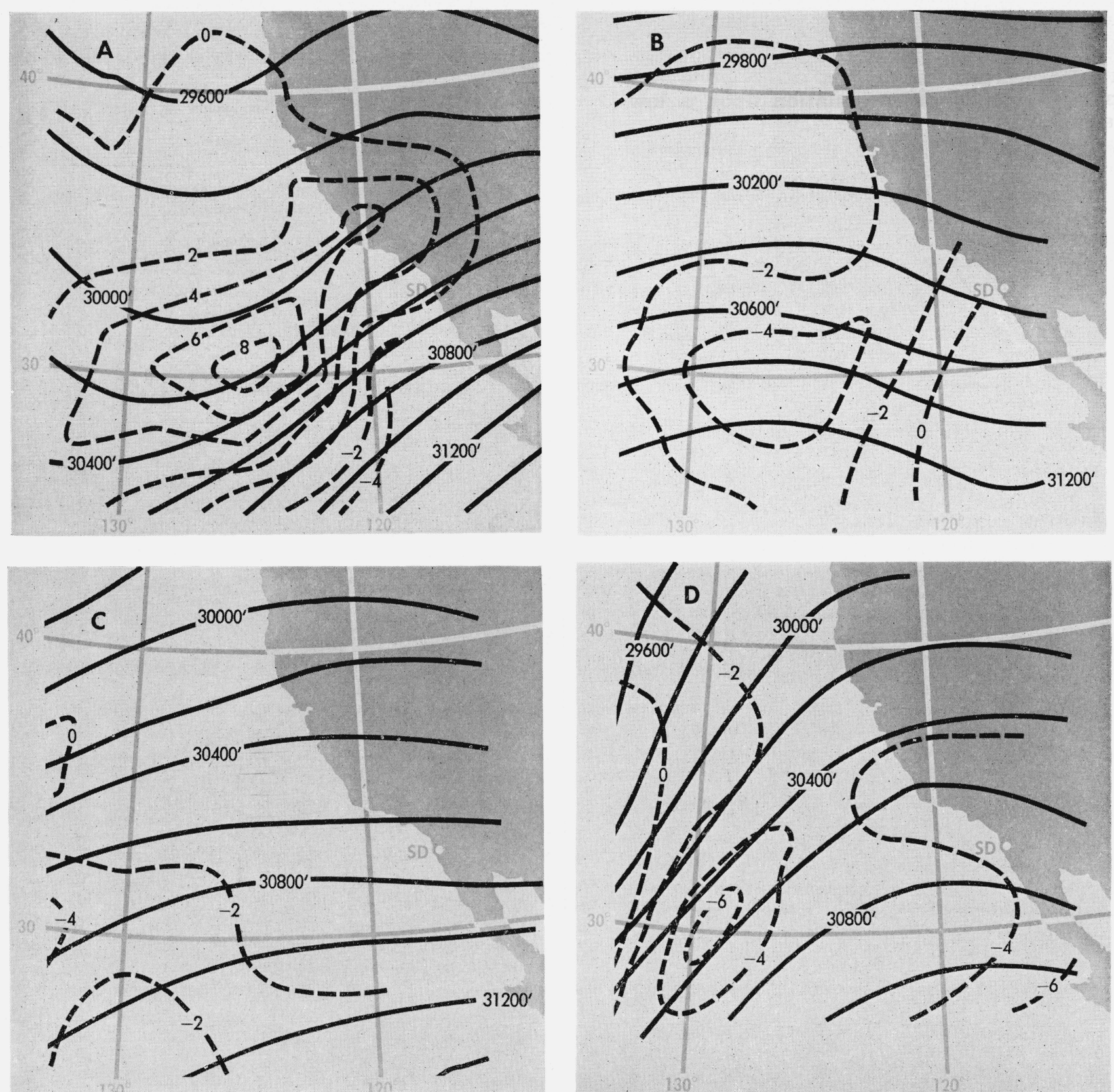

FIGURE 3. Three hundred millibar pressure-height contours (solid) and isopleths of relative vorticity (dashed) in units of $10^{-5} \mathrm{t}^{-1}$ at 1600 P.s.t. for (a) 16 March (b) 17 March (c) 18 March (d) 19 March.

siderations (app. B), positive vorticity advection implies negative vertical velocities. Figure 4 shows $\nabla \cdot V 300 \mathrm{mb}$ as a function of time compared with a series of refractive index profiles derived from the Los Angeles radiosonde observations. The functional relationship between the scattering angle $\theta$ and the refractive gradients has been derived in appendix A. Figure 5 shows the scattering angle for the grazing ray and height of the common volume for the Santa Barbara link during the 3-day period. The upper curve shows the signal level as a function of the scattering angle normalized for a standard atmosphere. The second curve is the radio field strength for the Santa Barbara $X$-band link for the period 0800 to 1600 P.s.t., 19 March. Radio data were not taken the previous davs.

On March 26 there was an anomalous $20-\mathrm{db}$ enhancement of signal level during a 3-hr period near noon that appeared unexplainable on the basis of radiosonde data or by vertical velocities inferred from the upper-troposphere divergence field. The refractive index profiles reduced from Los Angeles radiosonde observations for 0400 P.s.t. and 1600 P.s.t. show an elevated layer near $4,000 \mathrm{ft}$ (fig. 6) 

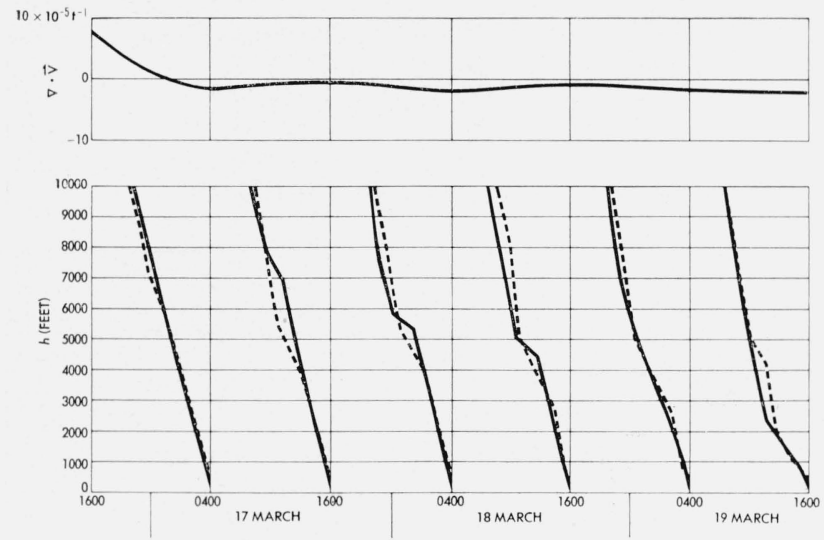

Figure 4. Horizontal velocity divergence at the 300-mb pressure level (upper graph) and Los Angeles and San Diego refractive index profiles for the period 16 to 19 March 1958.
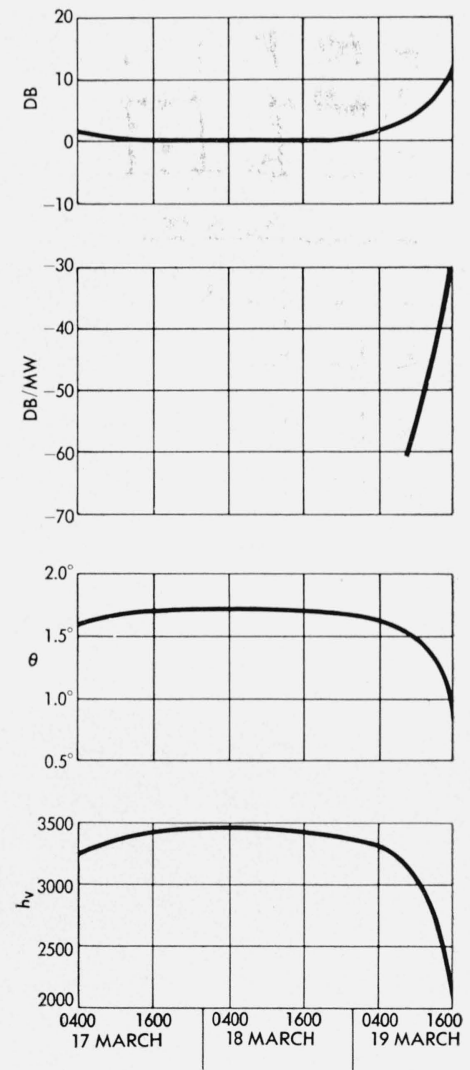

Figure 5. Upper curve indicates power depending upon scattering angle, $\theta$, for the period 17 to 19 March 1958. Second curve shows the received power in decibels per milliwatt for Santa Barbara X band. The lower two curves show the height of the scattering volume, $\mathrm{h}_{\mathrm{v}}$, and the scatiering angle, $\theta$, during the same period.

and computations of $\nabla \cdot V$ made at $300 \mathrm{mb}$ (upper curve fig. 6) indicate a continuous reduction in stability taking place. A series of isobaric and streamline southern California sectional charts were analyzed at hourly intervals for the day. Figure 7 shows the map series and includes the large-scale
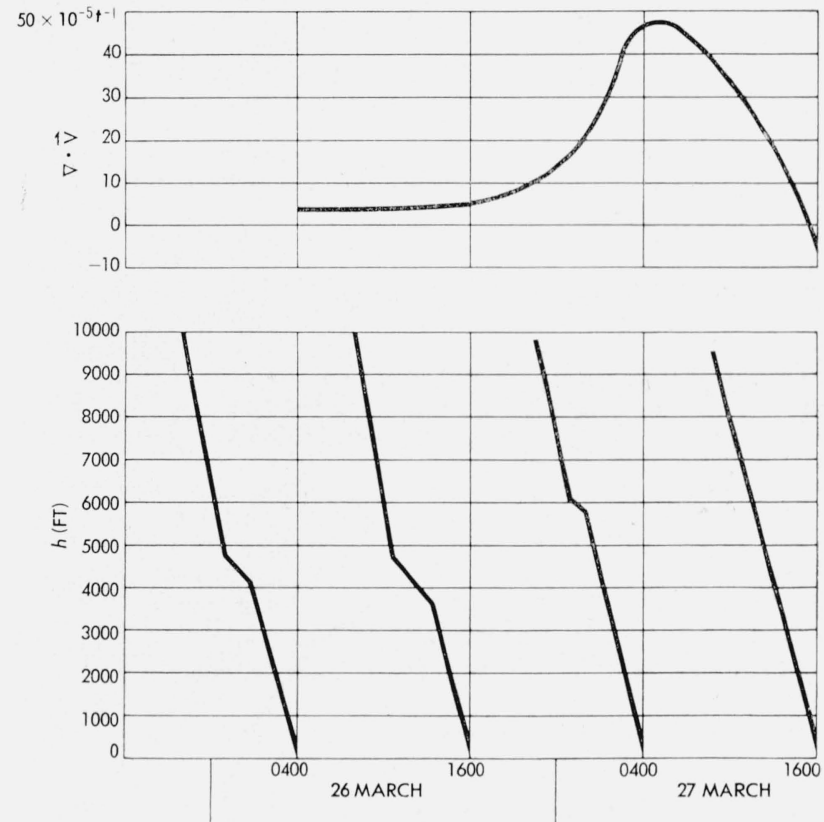

Figure 6. Refractive index profiles reduced from Los Angeles rabiosonde and (upper curve) 300-mb velocity divergence for 26 to 27 March 1958.

synoptic chart for 1000 P.s.t. Figure 8 is the Santa Barbara $X$-band signal for the day. From figure 7, at 0900 P.s.t., a cyclonic eddy with two centers northwest of San Diego lay across the Santa Barbara radio link. Streamlines indicate convergence and consequent upwelling of the surface air in the convergent areas. By 1100 P.s.t. the cyclonic eddy had weakened, due to the onset of a sea breeze and subsequent low-level divergence and subsidence of air over the Catalina channel. The 1200 P.s.t. map shows only a weak remnant of a zone of convergence south of Point Mugu and signals have risen $20 \mathrm{db}$. By 1300 P.s.t. offshore convergence had ceased entirely and signals had reached a peak level for the day shortly before 1300 P.s.t. The 1300 P.s.t. map indicates a strengthening of the pressure trough north of Point Mugu with a tendency for the trough to extend seaward. Signal levels started to fall after 1300 P.s.t. By 1500 P.s.t., the convergence zone had undergone strong redevelopment across the Santa Barbara link and signal levels had fallen about $22 \mathrm{db}$ from the peak level.

\subsection{Signal Level and Upper-Tropospheric Divergence}

During the period 26-28 March inclusive, the radio links were in operation from 0800 P.s.t. to 1600 P.s.t. daily. The weather regime was dominated by an upper-level cyclone which developed off the northwest coast of California and moved southeastward across the radio link. A series of $500-\mathrm{mb}$ height contour charts drawn at 12 -hr intervals during 

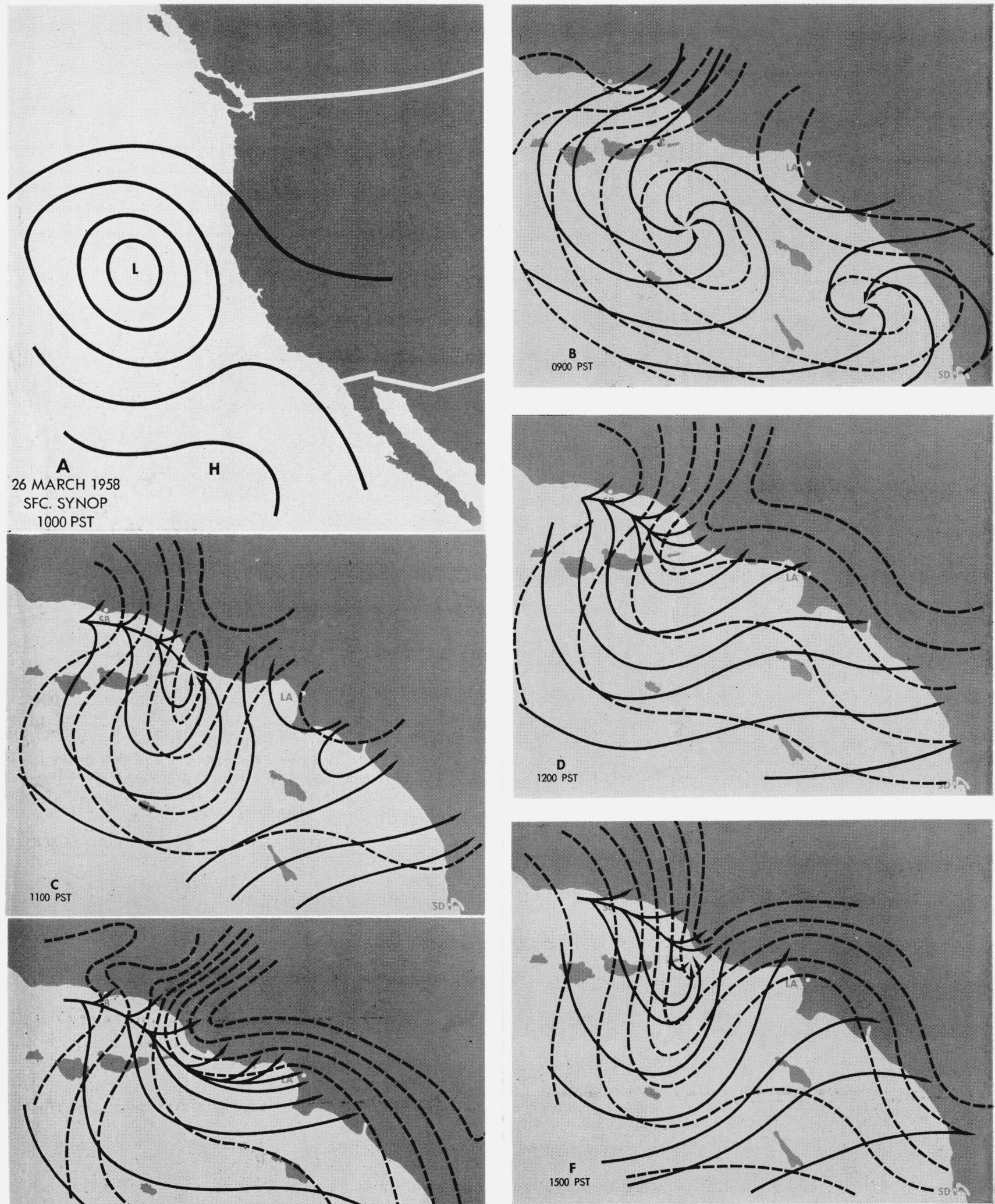

Figure 7. (A) Surface synoptic chart 1000 P.s.t. 26 March 1958. $(B-F)$ Sea level isobaric and streamline maps for southern California coastal waters during 26 March 1958. Dashed curves-are isobars, solid arrows indicate surface wind streamlines. 


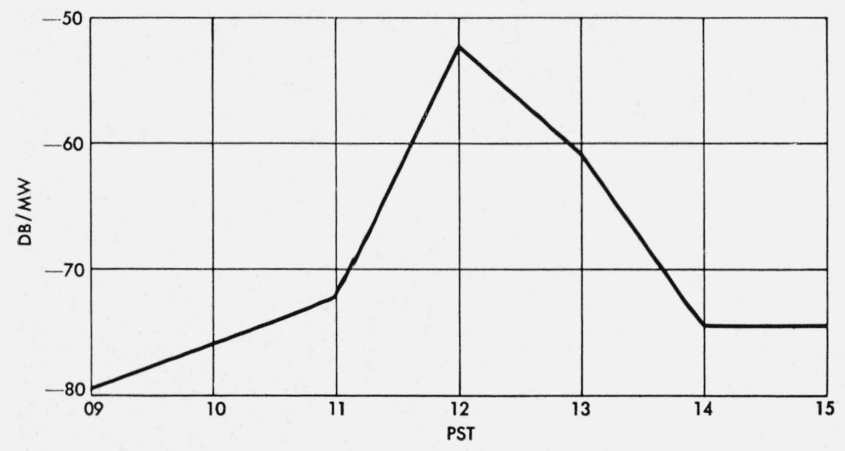

Figure 8. Santa Barbara X-band signal level for 26 March 1958 .

the period are shown in figure 9. The isopleths are contours of the height of the 500-mb pressure surface drawn for $200-\mathrm{ft}$ intervals. The velocity divergence along the streamlines $5^{\circ}$ upstream from the operating area was computed for each map during the period. Figure 10 is a graph of the velocity divergence at $500 \mathrm{mb}$ and average signal level received from the Santa Barbara $L$-band and Point Mugu $X$-band transmitters, both plotted as a function of time. The signal levels are average values for the periods $0800-1000$ P.s.t., and 1400 to 1600 P.s.t. plotted at 0900 and 1500 P.s.t., respectively, and connected with a smooth curve. Solid portions of the curve are for hours of radio transmission. The dashed curves are extrapolations of the solid curve slopes. Unfortunately, periods of radio silence were longer than transmission periods so that one can only assume that the signal strength curves are reasonably close to those extrapolated. Although it would be impossible to predict absolute signal level, the correlation between the sign and relative magnitudes of the time derivatives of the curves is good.

Computations of vorticity advection were made at the $500-\mathrm{mb}$ pressure level for all days when there was a reasonably good sample of radio data and at the 300-mb level for periods of particular meteorological interest. Signal levels for the 2-hr period nearest map time were averaged for the conditions of positive and negative vorticity advection (positive and negative vertical velocities). Table 1 gives these

TABLE 1. Average values of signal level for conditions of positive and negative vertical wind velocities

\begin{tabular}{|c|c|c|c|c|}
\hline \multirow{2}{*}{$\begin{array}{c}\text { Pt. Magu and Santa Barbara } \\
\text { transmitters }\end{array}$} & \multicolumn{2}{|c|}{$w>0$} & \multicolumn{2}{|c|}{$w<0$} \\
\hline & $500 \mathrm{mb}$ & $300 \mathrm{mb}$ & $500 \mathrm{mb}$ & $300 \mathrm{mb}$ \\
\hline $\begin{array}{l}B_{x-1} \\
R L \\
M_{x} \\
M_{L-2}\end{array}$ & $\begin{array}{l}-69.5 \\
-67.0 \\
-68.4 \\
-66.0\end{array}$ & $\begin{array}{l}-71.0 \\
-70.0 \\
-67.1 \\
-71.0\end{array}$ & $\begin{array}{l}-62.1 \\
-57.6 \\
-58.4 \\
-62.3\end{array}$ & $\begin{array}{l}-63.8 \\
-60.0 \\
-61.0 \\
-64.4\end{array}$ \\
\hline
\end{tabular}

average values of signal under the two conditions from a sample size of 33 at the $500-\mathrm{mb}$ level and 18 at the $300-\mathrm{mb}$ level. Both the $X$-and $L$-band signal levels are significantly higher under conditions of subsidence with no apparent frequency dependency to be noted.

\section{Results and Conclusions}

Other surface streamline charts and upper-tropospheric synoptic series were analyzed, but to present the results here would be repetitious and redundant. All concurrent meteorological and radio signal level analyses showed essentially the same features as indicated in the previous sections.

During the NEL experiment, sea level values of refractive index were essentially invariant with time, yet signal levels ranged over approximately $60 \mathrm{db}$. It seems clear that for over-water paths, short-term propagation characteristics are independent of surface refractivity.

Nonducting low-level refractive layers influence transhorizon fields in two ways: (1) refraction and subsequent decrease of scattering angle, and (2) partial reflection at the interfaces of superrefractive layers as the layers approach critical reflection heights and intensities. The second effect dominates for layers below about 2,000 ft. For example, if after Batchelor [7], a dielectric fluctuation spectrum of the form $E(K) \propto K^{-\frac{5}{3}}$ is accepted, then for narrow-beam propagation, the received power dependency upon scattering angle would be $\operatorname{Pr} \propto \theta / 2^{-\frac{1}{3}}$. Assuming standard propagation early in the morning of March 19, the Los Angeles sounding for 1600 P.s.t. March 19 (fig. 4) would indicate a lessening of scattering angle sufficient to cause a 12.7 -db rise in signal level (fig. $5)$. The actual rise in signal was approximately 35 db. In order to account for such a signal rise by refraction, the scattering angle would have had to decrease by nearly one-sixth. It is unlikely that the overwater refractive gradients were sufficiently greater than those shown by the Los Angeles radiosonde to account for this much refraction. One is forced to conclude that the partial reflection mechanism becomes more important for transhorizon propagation than scattering when relatively low layers are present. This suggests that the refractive index climatology charts for oceanic areas [15] could be useful for determining the distribution of dominant modes of transhorizon propagation.

The short term prediction of change of radio range for oceanic areas becomes greatly simplified if the problem can be looked upon as one concerning only changes in thermal stability. Except for coastal regions where continental air may undergo rapid modification, refractive layering and thermal stability over the oceans are principally functions of vertical wind velocities. Techniques for computing exactly or making qualitative estimates of the vertical wind velocities similar to the one outlined in appendix B are familiar to meteorologists. It is likely that satisfactory estimates of radio scatter propagation ranges can be made indirectly from regularly available weather charts. 

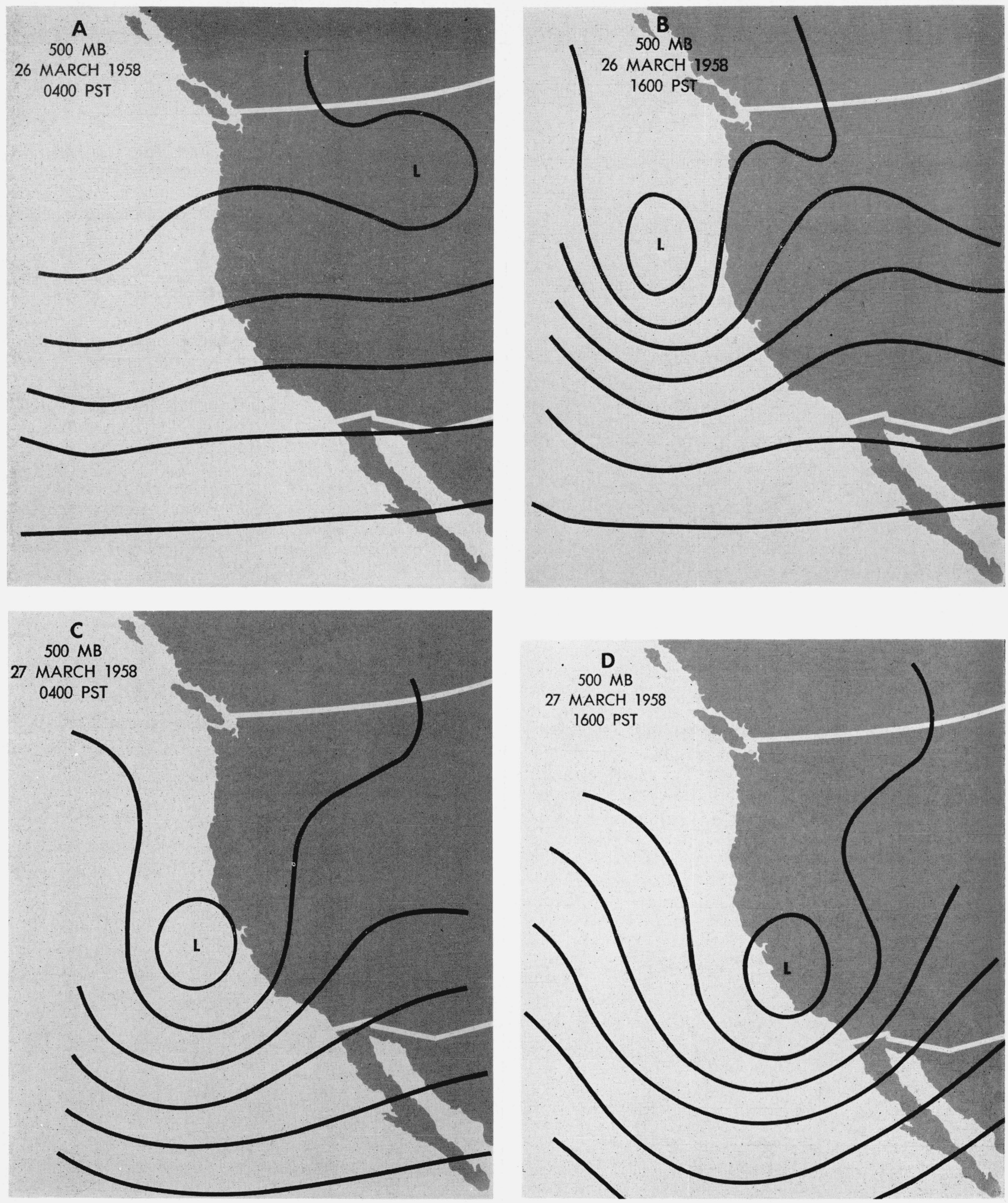

Figure 9. Five hundred millibar pressure-surface contour charts at 12-hr intervals from 0400 P.s.t. 26 March to 0400 P.s.t. 28 March. 


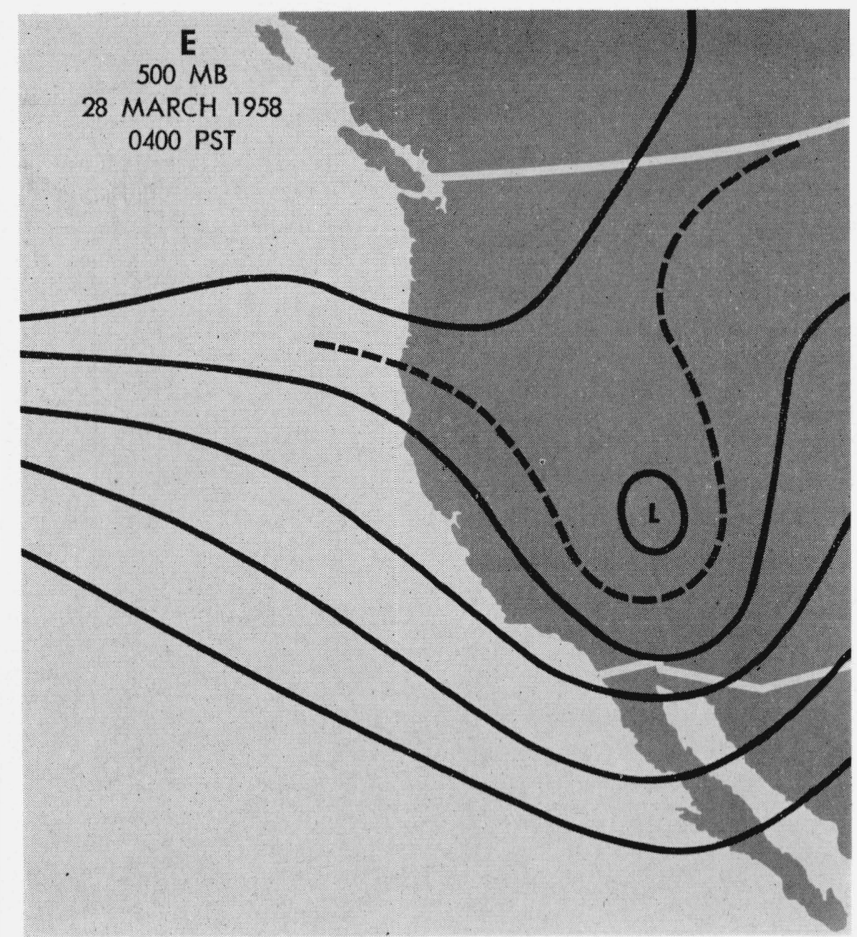

Figure 9.-Continued.

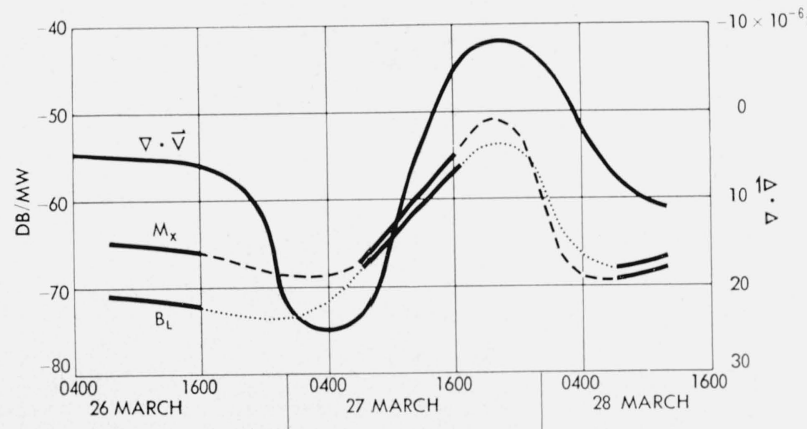

Figure 10. Santa Barbara L-band and Point Mugu X-band signal levels and 500-mb velocity divergence for 0400 P.s.t. for 26 March to 1600 P.s.t. 28 March inclusive.

Dashed signal level curves are extrapolated values for periods of radio silence.

\section{Appendix A: Scattering Angle and Height of the Common Scattering Volume}

The scattering volume is the portion of atmosphere common to both transmitting and receiving antenna beams. The acute angle at the beam intersection is called the scattering angle. It is possible to compute the height of the base of the scattering volume and the magnitude of the scattering angle from the gross refractive index profile reduced from radiosonde or refractometer data.
The atmospheric model (fig. 11) assumes horizontal homogeneity of a refractive layer for the distance that a ray segment remains within the layer. The number of refractive layers may vary with distance over the scattering link. The horizontal distribution of vertical refractive index gradients may be interpolated from soundings taken near either end of the radio link.

In figure 11, unprimed quantities refer to parameters between the transmitting (receiving) antenna and the beam intersection; primed quantities refer to parameters between the intersection and the receiving (transmitting) antenna. Symbol meanings are as follows:

$A=$ antenna heights,

$d_{0}=$ distance to the radio horizon,

$d_{i}=$ projection on the earth of the ray path in the $i$-th layer,

$D=$ distance between antennas,

$w_{i}=$ thickness of $i$-th layer,

$M=$ modified refractive index $=(n-1) \times 10^{6}$ $+0.048 h$,

$h_{v}=$ height of base of the common volume, $\theta=$ scattering angle.

Subscript $v$ refers to the refractive layer wherein the beams intersect. From figure 11:

$$
S=d_{v}+d_{v}^{\prime}=D-\sum_{0}^{v-1} d_{i}
$$

The angle which the ray makes with the local horizontal upon emerging from the $n$-th layer is given by

$$
d_{n}=10^{-3}\left[10^{+6} \alpha_{0}+2 \sum_{1}^{n} W_{i} \frac{d M}{d h} i\right]^{\frac{1}{2}} .
$$

For the case of grazing propagation $\alpha_{0}$ is the angle the ray makes with the horizon and is zero. For nongrazing propagation $\alpha_{\mathrm{o}}$ is the elevation angle of the antenna beam. Figure 12 is a graph of $2 W d M / d h$ as a function of $w$ and $\Delta M / 1,000 \mathrm{ft}$. Figure 13 is a nomographic solution of (A2). The incremental distances $d_{0}$ and $d_{1}$ can be easily determined from figure 14 , the solution of

$$
d=10^{3}\left[2 H\left(\frac{d M}{d h}\right)^{-1}\right]^{\frac{1}{2}} .
$$

The parameter $H$ is either antenna height or the depth of the surface-based refractive layer at each end of the link.

The incremental distances $d_{i}$ from $d_{2}$ to $d_{v-1}$ may be determined from figure 15 , a graphical solution of

$$
d_{i}=10^{6}\left(\alpha_{i}-\alpha_{i-1}\right)\left(\frac{d M}{d h}\right)^{-1} \text {. }
$$

$S$ may now be determined according to (A1). The remaining distances, $d_{v}$ and $d_{v}{ }^{\prime}$, are given by 


$$
d_{v}=\frac{\frac{S^{2}}{4}\left(\frac{d M}{d h}\right)_{v} 10^{-6}+S \alpha_{v-1}+\Delta W}{\frac{S}{2}\left(\frac{d M}{d h}\right)_{v} 10^{-6}+\alpha_{v-1}+\alpha_{v-1}^{\prime}}
$$

and

$$
d_{v}^{\prime}=S-d_{v}
$$

The height of the base of the common volume is now determined from

$$
h_{v}=d_{v}\left(\alpha_{v-1}+10^{6} \frac{d_{v}}{2} \frac{d M}{d h_{v}}\right)+\sum_{1}^{v-1} W_{i}
$$

and the scattering angle from

$$
\theta=\alpha_{v}+\alpha_{v}^{\prime}+10^{-6} S \frac{d M}{d h_{v}} .
$$

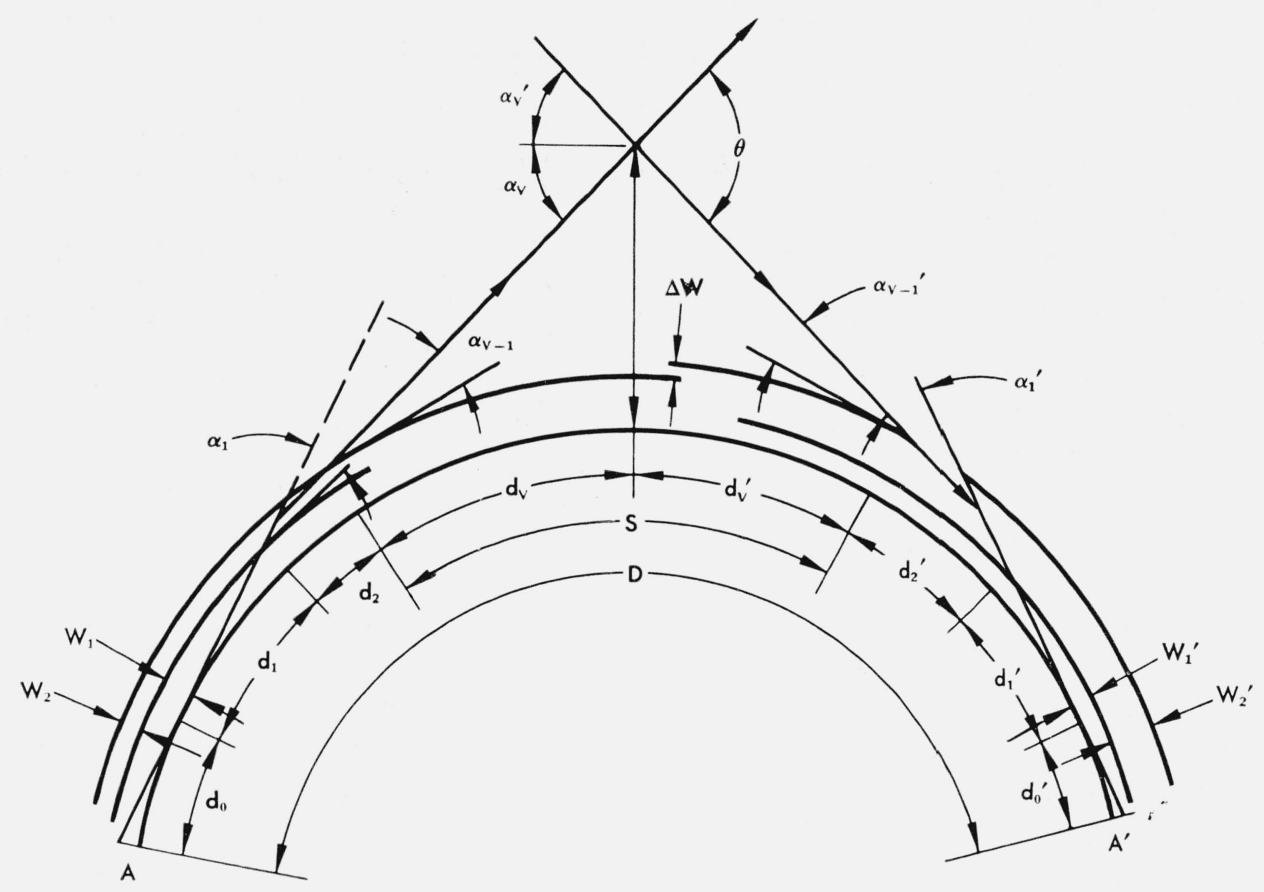

FIGURE 11. Schematic diagram of scatter propagation link with nonhomogeneous, layered atmosphere.

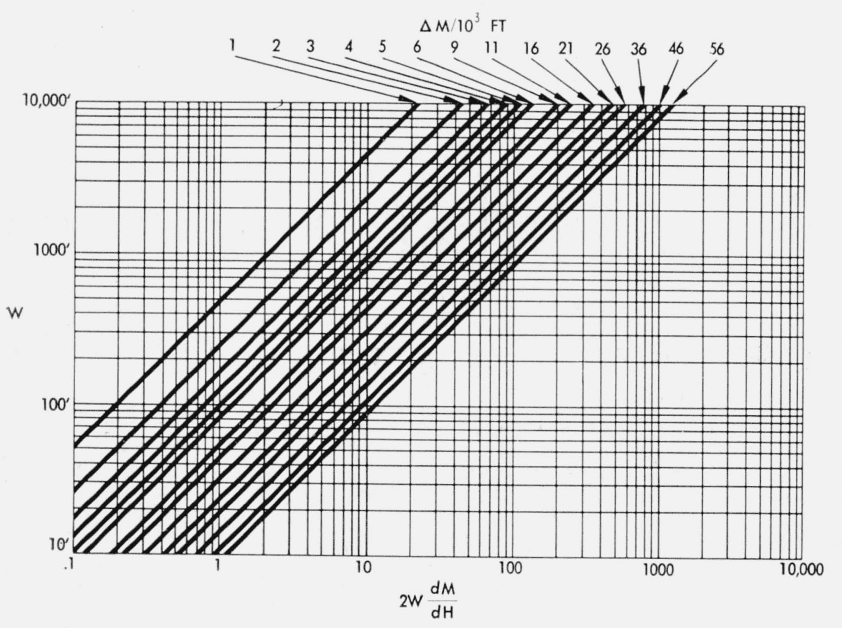

Figure 12. Nomogram of $2 \mathrm{w} \frac{\mathrm{dm}}{\mathrm{dh}}=\mathrm{f}\left(\mathrm{w}, \Delta \mathrm{M} / 10^{3} \mathrm{ft}\right)$.

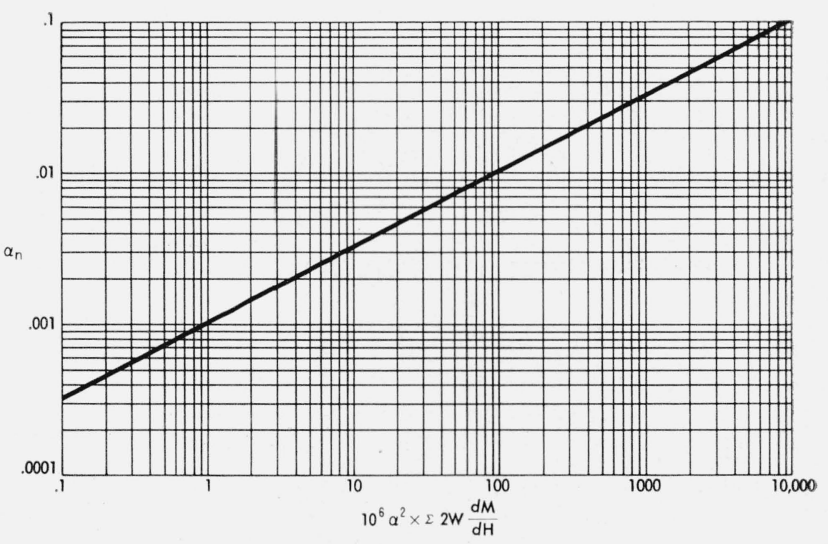

Figure 13. Nomogram of $\alpha_{\mathrm{n}}=\mathrm{f}\left(\mathrm{W}_{\mathrm{i}}, \Delta \mathrm{M} / 10^{3} \mathrm{ft}, \alpha_{\mathrm{o}}\right)$. 


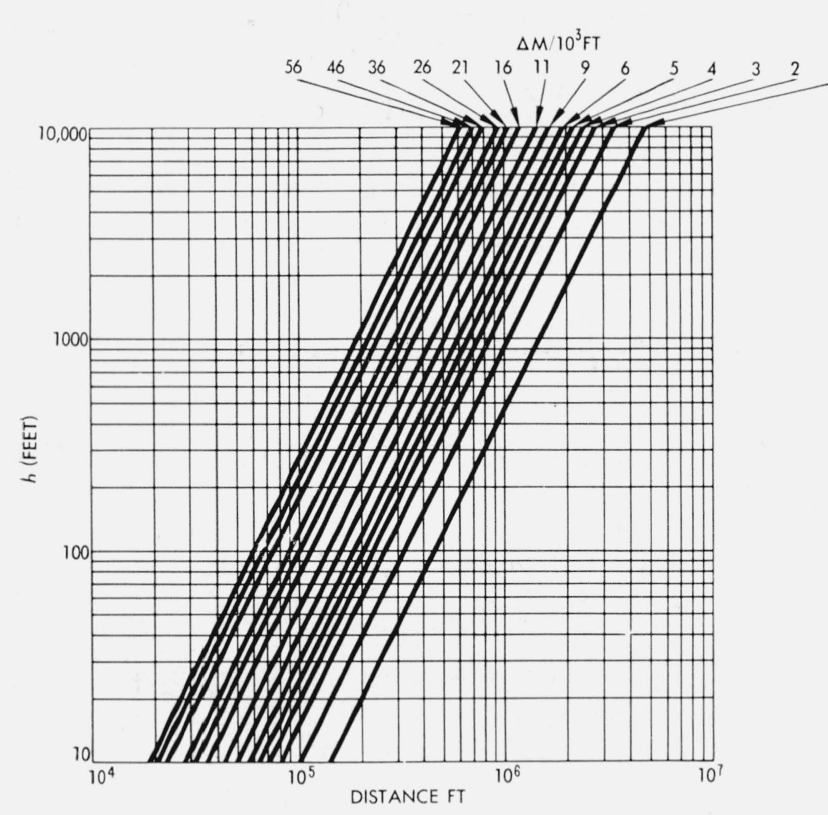

Figure 14. Nomogram of $\mathrm{d}=\mathrm{f}\left(\mathrm{H}, \Delta \mathrm{M} / 10^{3} \mathrm{ft}\right)$.

\section{Appendix B}

7.1. Method for Estimating the Relative Magnitude and Direction of the Vertical Component of the Wind

It may become necessary for the radio meteorologist to prepare operational propagation forecasts for areas where machine-computed vertical velocity charts are not available. The following simple method for obtaining a "better-than-guess" estimate of the vertical-motion field is suggested as a forecast aid.

The atmospheric model assumes the following conditions:

1. An equivalent barotropic level (or level of nondivergence) exists in the midtroposphere, usually at a lower altitude than the 500-mb surface and certainly below the $300-\mathrm{mb}$ surface.

2. The horizontal wind velocity divergence changes sign at the level of nondivergence (from here on "LND") on the vertical coordinate and at no other elevation.

3. The direction and magnitude of vertical velocity throughout the troposphere are the same in sign and proportional in magnitude to those at the LND.

4. Vertical velocities are nonexistent at the surface of the earth and the outer boundary of the atmosphere.

One form of the equation of continuity is

$$
\frac{\partial \rho}{\partial t}+\boldsymbol{V} \cdot \nabla \rho+\rho \nabla \cdot \boldsymbol{V}+\frac{\partial}{\partial Z}(\rho W)=0
$$

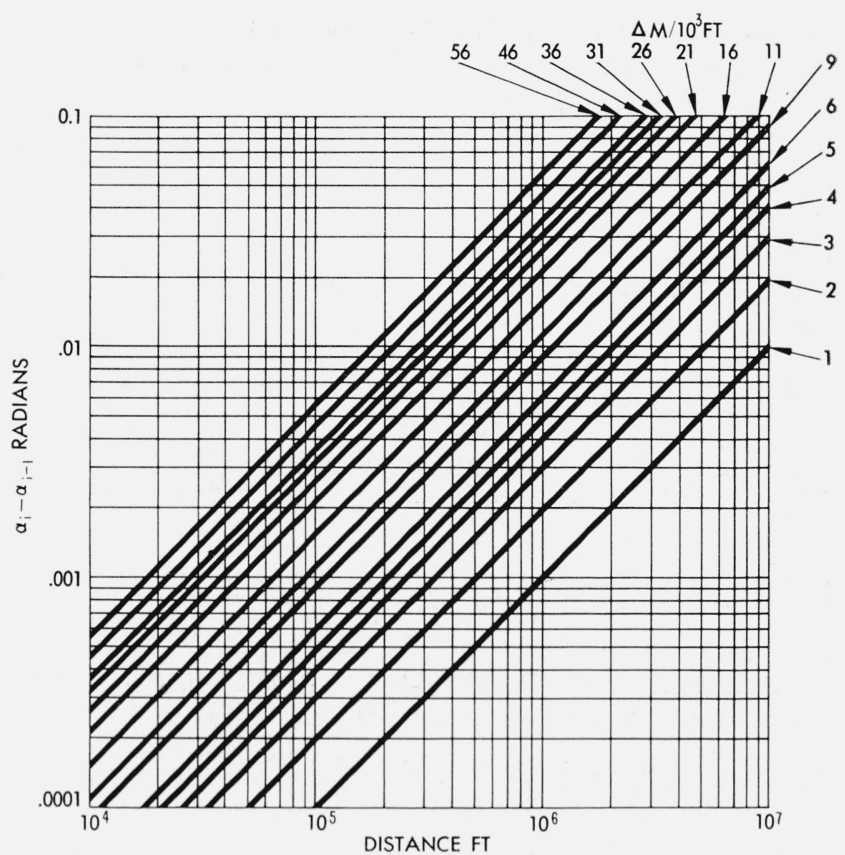

Figure 15. Nomogram of $\mathrm{d}_{\mathrm{i}}=\left(\alpha_{\mathrm{i}}-\alpha_{\mathrm{i}-1}\right)\left(10^{3} \Delta \mathrm{M}^{-1}\right)$.

where $\rho=$ density of the air, $\boldsymbol{V}=$ horizontal wind velocity vector, $\nabla=$ horizontal gradient operator, $Z=$ vertical coordinate, $W=$ vertical wind velocity. Integration of (B1) from the LND to the outer atmosphere results in

$(\rho W)_{\mathrm{LND}}=\int_{\mathrm{LND}}^{\infty} \boldsymbol{V} \cdot \nabla \rho d Z+\int_{\mathrm{LND}}^{\infty} \frac{\partial \rho}{\partial t} d Z+\int_{\mathrm{LND}}^{\infty} \rho \nabla \cdot \mathbf{V} d Z$

It is usually observed that $\partial \rho / \partial t \ll \rho \nabla \cdot \boldsymbol{V}$ so that under most circumstances (B2-b) may be disregarded. It is also observed that cold air advection below the tropopause is accompanied by warm air advection in the stratosphere. For such a case $(\mathrm{B} 2-\mathrm{a}) \rightarrow 0$. With these assumptions (B2) reduces to

$$
(W)_{\mathrm{LND}} \simeq \frac{1}{\rho} \int_{\mathrm{LND}}^{\infty} \rho \nabla \cdot \boldsymbol{V} d \mathrm{Z}
$$

Horizontal velocity divergence is defined as

$$
\nabla \cdot \boldsymbol{V}=\frac{\partial v}{\partial s}+v \frac{\partial \theta}{\partial n}
$$

in the natural coordinate system. Evaluation directly from the wind field is difficult to accomplish with any degree of accuracy. For this reason an alternate expression for the integrand of (B3) is sought. 
The vorticity equation is expressed

$$
\zeta^{-1} \frac{d \zeta}{d t}=-\nabla \cdot \mathbf{V}
$$

where $\zeta$ is the vertical component of the absolute vorticity. The substantial derivative equated to its partial components is

$$
\frac{d \zeta}{d t}=\frac{\partial \zeta}{\partial t}+V \frac{\partial \zeta}{\partial s}+W \frac{\partial \zeta}{\partial Z}
$$

where $s$ is along the streamlines. Substituting eq (B5) in (B3) we have

$$
\begin{aligned}
(W)_{\mathrm{LND}}=-\frac{1}{\rho}\left[\int_{\mathrm{LND}}^{\infty} \frac{\rho}{\zeta} \frac{\partial \zeta}{\partial t} d Z\right. & +\int_{\mathrm{LND}}^{\infty} \frac{\rho}{\zeta} v \frac{\partial \zeta}{\partial s} d Z \\
& \left.+\int_{\mathrm{LND}}^{\infty} \frac{\rho}{\zeta} W \frac{\partial \zeta}{\partial Z} d Z\right] .
\end{aligned}
$$

The first integral on the right becomes proportionately smaller with increasing height above the LND and at the $300-\mathrm{mb}$ level is generally quite small compared with the second integral. This is merely a statement that, in general, the winds blow through the pressure contours faster than the pressure contours are moving. The third integral is small because in the integral over the entire upper atmosphere the only contribution can pass through the lower boundary. Because the vertical velocity is several orders of magnitude smaller than horizontal components, the contribution cannot be great.

We can then write

$$
(W)_{\mathrm{LND}} \cong-\frac{1}{\rho} \int_{\mathrm{LND}}^{\infty} \frac{\rho}{\zeta} V \frac{\partial \zeta}{\partial s} d Z
$$

The absolute vorticity, $\zeta=f+\zeta_{r}$, and its gradient $\partial \zeta / \partial s=\partial f / \partial s+\partial \zeta_{r} / \partial s$ may be evaluated directly from a constant pressure chart. The gradient of the Coriolis term, $\partial \zeta / \partial s$ is, under most circumstances, at least an order of magnitude less than the gradient of relative vorticity, $\partial \zeta_{r} / \partial s$.

The magnitude of the vertical component of relative vorticity is $\zeta_{r}=(\partial v / \partial x)-(\partial u / \partial y)$ Substituting in eq (B7) we have finally

$$
(W)_{\mathrm{LND}}=-\frac{1}{\rho} \int_{\mathrm{LND}}^{\infty} V \frac{\rho}{\zeta} \frac{\partial}{\partial s}\left(\frac{\partial v}{\partial x}-\frac{\partial u}{\partial y}\right) d Z
$$

If the assumptions stated at first are reasonable then the sign of

$$
(W)_{\mathrm{LND}}=-\operatorname{sign} \text { of }\left[\frac{\partial}{\partial s}\left(\frac{\partial v}{\partial x}-\frac{\partial u}{\partial y}\right)\right]_{h} \quad h>h_{\mathrm{LND}}
$$

Further, the $300-\mathrm{mb}$ level is near the jetstream (region of maximum winds) and (with somewhat less certainty) one can also say

relative magnitude $\left(W_{\mathrm{LND}}=\right.$ relative magnitude of

$$
\frac{V}{\zeta} \frac{\partial}{\partial s}\left(\frac{\partial v}{\partial x}-\frac{\partial u}{\partial y}\right) 300 \mathrm{mb}
$$

\subsection{Computation of $\zeta_{r}$}

The computation of relative vorticity on a constant pressure chart is greatly simplified if one makes use of the geostrophic wind approximation

$$
U_{o}=-\frac{g}{f} \frac{d h}{d y}, \quad V_{g}:=\frac{g}{f} \frac{d h}{d x} .
$$

The finite difference equation for the relative vorticity is then

$$
\zeta_{r}=\frac{g}{f}\left(\frac{\Delta h}{(\Delta x)^{2}}+\frac{\Delta h}{(\Delta y)^{2}}\right)
$$

The vorticity field is computed by reading the constant pressure surface height at evenly spaced grid points and carrying out the computation

$$
\zeta_{r}=\frac{g}{f D^{2}}\left(H_{1}+H_{2}+H_{3}+H_{4}-4 H_{5}\right)
$$

where $f$ is the Coriolis parameter (twice the angular velocity of the earth times the sine of the latitude), $g$ the acceleration of gravity, $D$ is the grid spacing, and $H_{1}, H_{2}, H_{3}, H_{4}, H_{5}$ are the pressure surface heights at the points $P_{1} \ldots P_{5}$ as shown in figure 16. The computed vorticity is the average value for the grid space and is plotted at $P_{5}$.

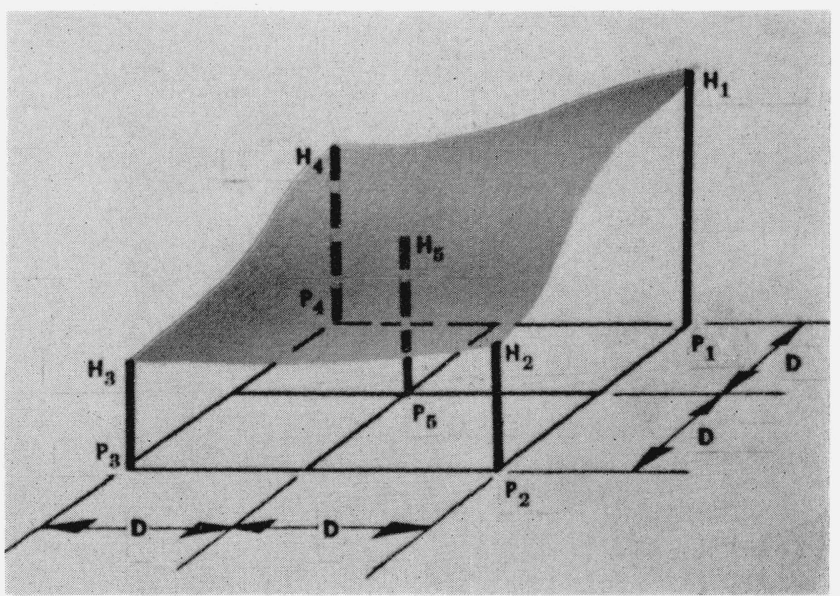

FIGURE 16. Schematic of grid used to compute relative vorticity on a constant pressure surface.

In this report the relative vorticity was computed using a grid size of $5^{\circ}$ latitude for a grid centered over Catalina Island and another grid $5^{\circ}$ latitude upwind. The vorticity gradient was assumed to be the finite difference between $\zeta_{r}$ at the two positions divided by the $5^{\circ}$ latitude distance between points. 


\section{References}

[1] B. R. Bean, Some meteorological effects on scattered radio waves, IRE Trans. CS-4, 32 (1956).

[2] J. B. Smyth and others, Atmospheric refraction; a preliminary quantitative investigation, Navy Radio and Sound Lab. Rept. WP-17 (28 Dec. 1944).

[3] J. B. Smyth and L. G. Trolese, Propagation of radio waves in the lower troposphere, Proc. I.R.E. 35, 1198 (1947).

[4] E. E. Gossard and L. J. Anderson, The effect of superrefractive layers on 50-5,000 Mc non-optical fields, IRE Trans. AP-4, 175 (1956)

[5] H. G. Booker and W. E. Gordon, A theory of radio scattering in the troposphere, Proc. I.R.E. 38, 401 (1950)

[6] F. Villars and V. F. Weisskopf, The scattering of electromagnetic waves by turbulent atmospheric fluctuations, Phys. Rev. 94, 232 (1954).

[7] G. K. Batchelor, The scattering of radio waves in the atmosphere by turbulent fluctuations in refractive index, Cornell Univ., School of Elec. Eng., Tech. Rept. 26 (15 Sepı. 1955).

[8] J. R. Bauer, The suggested role of stratified elevated layers in transhorizon short-wave radio propagation, Mass. Inst. Technol., Lincoln Lab., Tech. Rept. 124 (24 Sept. 1956).
[9] H. T. Frijs and others, A reflection theory for propagation beyond the horizon, Bell System Tech. J. 36, 627 (May 1957).

[10] D. L. Ringwalt and others, Measurements of Mc/s scatter propagation as function of meteorology (letter submitted to IRE for PGAP series).

[11] P. Misme, The correlation between the electric field at a great distance and a new radio-meteorological parameter, IRE Trans. AP-6, 289 (1958)

[12] W. F. Moler and W. A. Arvola, Vertical motion in the atmosphere and its effect on VHF radio signal strength, Trans. Am. Geophys. Union 37, 399 (1956).

[13] E. E. Gossard, Power spectra of temperature, humidity and refractive index from aircraft and tethered balloon measurements (to be published).

[14] W. E. Gordon, Radio scattering in the troposphere, Proc. I.R.E. 43, 23 (1955).

[15] J. Michaelis and E. E. Gossard, Distribution of refractive layers over the North Pacific and Arctic, Navy Electron. Lab. Report 841 (18 Apr. 1958).

Boulder, Colo.

(Paper 64D1-42) 